\title{
Nuclear KLLN expression associates with improved relapse-free survival for prostate carcinoma
}

\author{
Yu Wang ${ }^{1}$, Andres Roma ${ }^{2}$, Rosalie Nolley ${ }^{3}$, Fadi Abdul-Karim ${ }^{2}$, Donna M Peehl ${ }^{3}$ and \\ Charis Eng ${ }^{1,4,5,6,7}$ \\ ${ }^{1}$ Genomic Medicine Institute ${ }^{2}$ Department of Anatomic Pathology, Cleveland Clinic, Cleveland, Ohio 44195, USA \\ ${ }^{3}$ Department of Urology, Stanford University School of Medicine, Stanford, California 94305, USA \\ ${ }^{4}$ Taussig Cancer Institute ${ }^{5}$ Stanley Shalom Zielony Institute for Nursing Excellence, Cleveland Clinic, Cleveland, \\ Ohio 44195, USA \\ ${ }^{6}$ Department of Genetics and Genome Sciences ${ }^{7}$ CASE Comprehensive Cancer Center, Case Western Reserve \\ University School of Medicine, Cleveland, Ohio 44106, USA
}

\author{
Correspondence \\ should be addressed \\ to C Eng \\ Email \\ engc@ccf.org
}

\begin{abstract}
Men with organ-confined prostate cancer ( $\mathrm{CaP}$ ) are often treated with radical prostatectomy. Despite similar postoperative characteristics, a significant proportion of men with an intermediate risk of progression experience prostate-specific antigen (PSA)-defined failure, while others have relapse-free survival (RFS). Additional prognostic markers are needed to predict the outcome of these patients. KLLN is a transcription factor that regulates the cell cycle and induces apoptosis in cancer cells. We have shown that $K L L N$ is an androgenregulated gene and that loss of $K L L N$ expression in primary $\mathrm{CaP}$ is associated with high Gleason score. In this retrospective study, we evaluated $K L L N$ expression in the high-grade malignancy components from 109 men with intermediate risk CaP. Patients with nuclear KLLN-negative tumors had significantly higher preoperative serum PSA levels (12.24 \pm $2.37 \mathrm{ng} / \mathrm{ml})$ and larger tumor volumes $\left(4.61 \pm 0.71 \mathrm{~cm}^{3}\right)$ compared with nuclear KLLN-positive patients $\left(8.35 \pm 2.45 \mathrm{ng} / \mathrm{ml}, P=0.03\right.$, and $2.66 \pm 0.51 \mathrm{~cm}^{3}, P<0.0001$, respectively). None of the nuclear KLLN-positive tumors had capsular penetration, whereas $34 \%$ of nuclear KLLNnegative tumors $(P=0.004)$ had capsular penetration. Maintaining KLLN expression in tumor nuclei, but not in cytoplasm or stroma, associated with improved RFS after surgery $(P=0.002)$. Only $7 \%$ of patients with nuclear KLLN-positive tumors had tumor recurrence, while $60 \%$ of patients in the KLLN-negative group developed PSA-defined failure with median relapse time of 6.6 months $(P=0.0003)$. Our data suggest that KLLN expression may be used as a prognostic marker to predict outcome for intermediate risk patients, which could provide useful information for postoperative management.
\end{abstract}

\author{
Key Words \\ - KLLN \\ - prostate cancer \\ - PSA failure \\ - relapse-free survival \\ - radical prostatectomy
}

Endocrine-Related Cancer (2014) 21, 579-586

\section{Introduction}

Prostate cancer $(\mathrm{CaP})$ was diagnosed in $\sim 239000$ men in the United States and accounted for more than 29000 deaths in 2013 (Siegel et al. 2013). Most men diagnosed in the prostate-specific antigen (PSA) era have favorable disease characteristics and progression-free survival. Approximately, $35 \%$ of $\mathrm{CaP}$ patients have intermediate 
risk prostate cancer (IRPC) (D'Amico et al. 1998a, DeSantis et al. 2013). According to risk classification, the intermediaterisk group includes those with stage $\mathrm{T} 2 \mathrm{~b}-\mathrm{T} 2 \mathrm{c}$ tumors, PSA between 10 and $20 \mathrm{ng} / \mathrm{ml}$, or Gleason score 7 (D'Amico et al. 1998b). After radical prostatectomy (RP), 65-80\% of patients with IRPC had 5-year relapse-free survival (RFS) (D'Amico et al. 1998b,c,d, Meng et al. 2005, Park et al. 2005). In contrast, other IRPC patients with similar tumor characteristics have PSA-defined failure and metastasis after the same initial treatment, which uniformly lead to cancer-specific death. Currently, it remains a challenge to accurately predict oncologic outcomes for IRPC patients, especially for those with high Gleason grade lesions. Therefore, a reliable preoperative biomarker is urgently needed, which not only provides aggressive therapy for patients who will have recurrent tumor, but also prevents overtreatment in patients who do not relapse.

$K L L N$ (KILLIN) is a newly identified gene located in 10q23.31 (Cho \& Liang 2008). KLLN and tumor suppressor phosphatase and tensin homolog (PTEN) share the same promoter regions but are transcribed in the opposite direction (Cho \& Liang 2008, Bennett et al. 2011). KLLN is a DNA-binding protein that regulates cell cycle and apoptosis in diverse types of cancer cells (Cho \& Liang 2008, Wang et al. 2013a,b). Our previous studies have shown that KLLN functions as a tumor suppressor and transcription factor mainly localized to the cell nucleus (Wang et al. 2013a,b). In CaP cell lines, KLLN and androgen receptor (AR) negatively regulate each other at the transcriptional levels, resulting in a negative feedback loop (Wang et al. 2013b). Overexpression of KLLN suppresses AR expression and decreases PSA levels in CaP cells (Wang et al. 2013b). We have also shown that loss of KLLN expression in CaP associates with high Gleason score, suggesting that KLLN might be used as a potential prognostic marker for risk management (Wang et al. $2013 b$ ). In this study, we sought to analyze KLLN expression as a marker to predict clinical outcomes in patients with intermediate Gleason score CaP.

\section{Materials and methods}

\section{Patients and human tissues}

Paraffin-embedded RP specimens were collected at Stanford University Hospital. Consent had been obtained from each patient after full explanation of the purpose and nature of all procedures. Patients were identified with intermediate risk as per the National Comprehensive
Cancer Network (NCCN) standard. For this study, tissue sections containing high-grade (Gleason grades 4 or 5 ) cancer were selected from 109 patients who had Gleason score 7 peripheral zone CaP, containing $30-70 \%$ Gleason grades $4 / 5$. We excluded patients with tumor at clinical stage of T2c and higher. The percentage of Gleason grade $4 / 5$, preoperative serum PSA, weight of prostate, volume of index (largest) and secondary tumors, capsular penetration, surgical margin (SM), and seminal vesicle invasion (SVI) were recorded. We excluded patients who received neoadjuvant therapy before $\mathrm{RP}$ or adjuvant treatment after RP. Follow-up after RP followed American Urological Association guideline, including measurements of serum PSA every 6 months for 5 years and annually thereafter. The Cleveland Clinic Institutional Review Board for Human Subjects Protection approved the protocol for analyzing anonymized tissue. We carried out in silico study analysis of $343 \mathrm{CaP}$ tissue samples from The Cancer Genome Atlas (TCGA) Data Portal. We chose 119 patients who had Gleason $7 \mathrm{CaP}$ and analyzed for KLLN/PTEN copy number variation and mRNA expression analysis.

\section{Immunohistochemistry and quantification}

Tissue sections were cut at $5 \mu \mathrm{m}$, deparaffinized in xylene, and rehydrated in a series of graded ethanol. Antigen retrieval was carried out with $0.01 \mathrm{M}$ citrate buffer at $\mathrm{pH} 6.0$ for $20 \mathrm{~min}$ in a $121^{\circ} \mathrm{C}$ pressure chamber. The slides were allowed to cool for another $30 \mathrm{~min}$, followed by sequential rinsing in PBS-T $(0.01 \%$ Triton$\mathrm{X})$. Endogenous peroxidase activity was quenched by incubating in PBS-T containing 5\% hydrogen peroxide. Each incubation step was carried out at room temperature and was followed by three sequential washes in PBS-T. The sections were incubated in rabbit polyclonal anti-KLLN antibody (Abgent, San Diego, CA, USA) at dilution of 1:100 in PBS-T containing 5\% goat serum albumin, followed by incubations with biotinylated secondary antibody for $1 \mathrm{~h}$, peroxidase-labeled streptavidin (Vectastain system, Vector, Burlingame, CA, USA) for $1 \mathrm{~h}$, and diaminobenzidine-substrate for peroxidase-based immunohistochemistry (Cardassian DAB Chromogen, Biocare Medical, Concord, CA, USA). The slides were counterstained with hematoxylin (Vector) and mounted. W Y and two pathologists, $\mathrm{A}$ R and $\mathrm{F}$ A-K, independently evaluated the KLLN staining in Gleason grade 4 and 5 malignancies, blinded to outcomes. Positive KLLN staining in tumor cell nucleus, tumor cell cytoplasm, and adjacent stromal cells were recorded. The loss of

Published by Bioscientifica Ltd 
KLLN expression in nucleus, cytoplasm, or stroma was defined by negative staining in all cells at the prospective locations.

\section{Statistical analyses}

SVI, capsular penetration, positive SM, and KLLN expression between two groups were compared using the $\chi^{2}$ test. The differences in PSA levels, tumor volumes, prostate weight, and follow-up time between two groups were evaluated using unpaired t-test. Spearman's rank correlation coefficient was used to analyze pairwise correlation between patient characteristics. Variables associated with tumor recurrence were analyzed by univariate and multivariate logistic regression. PSAdefined failure was defined as the PSA nadir $+2 \mathrm{ng} / \mathrm{ml}$ according to the Phoenix criteria. RFSs were calculated to the event using the Kaplan-Meier method. Statistical analyses were performed using Prism version 6.0 (GraphPad Software, La Jolla, CA, USA) and SPSS statistical software package version 13.0 (SPSS, Inc.).

\section{Results}

\section{Patient characteristics}

This series had patients with a median age of $64 \pm 6.1$ years, median preoperative serum PSA level of $8.7 \pm$ $11.28 \mathrm{ng} / \mathrm{ml}$ and median follow-up of 2069 days (Table 1). The median tumor volume was $3.3 \pm 3.4 \mathrm{~cm}^{3}$. SVI presented in 26/109 (23.9\%) patients and 21/109 (19.3\%) patients had positive SM (Table 1 ). There were 33/109 (30\%) patient samples with capsular penetration (Table 1). Among these 109 patients, 50 (45.9\%) had RFS with the median $2251 \pm 161$ days of follow-up while 59 (54.1\%) experienced PSA-defined failure (Table 1). For patients with recurrent disease, the median time to relapse was $198 \pm 55$ days (Table 1 ).

\section{PSA-defined recurrence associated with loss of nuclear KLLN expression, SVI, and capsular penetration}

We first compared clinical and pathologic characteristics of patients with RFS $(n=50)$ and those with PSA-defined failure $(n=59)$. Both epithelial and stromal KLLN expressions were detected in Gleason grade 4 and/or 5 lesions (Fig. 1). We found 76\% of patients with RFS lost nuclear KLLN expression compared with $98 \%$ in patients who experienced recurrent disease $(P=0.0003)$ (Table 2$)$. In contrast, cancers from the relapse-free group and
Table 1 Clinical and pathological characteristics of overall cohort

\begin{tabular}{l} 
Characteristic \\
\hline Patient number \\
Age (years) \\
Mean \\
Median \\
Range \\
PSA (ng/ml) \\
Mean \\
Median \\
Range \\
Prostate weight $(\mathrm{g})^{\mathrm{a}}$ \\
Mean \\
Median \\
Range \\
Total tumor volume $\left(\mathrm{cm}^{3}\right)$ \\
Mean \\
Median \\
Range \\
Grade $4 / 5 \%{ }^{\mathrm{b}}$ \\
Mean \\
Median \\
Range \\
Capsular penetration $(\mathrm{cm})$ \\
Mean \\
Median \\
Range \\
Follow-up (days) \\
Mean \\
Median \\
Range \\
SVI \\
Positive margins \\
PSA failure \\
\\
\hline
\end{tabular}

\begin{tabular}{c}
\hline Value \\
\hline 109 \\
63.2 \\
64.0 \\
$48-73$ \\
11.8 \\
8.7 \\
$0.8-89.6$ \\
47.9 \\
43.0 \\
$25-96$ \\
4.4 \\
3.3 \\
$0.3-19.4$ \\
52.0 \\
50.0 \\
$30-70$ \\
0.5 \\
0 \\
$0-6$ \\
2094.8 \\
2069 \\
$363-4677$ \\
$26(23.9 \%)$ \\
$21(19.3 \%)$ \\
$59(54.1 \%)$ \\
\end{tabular}

SVI, seminal vesicle invasion.

${ }^{a}$ Weight of prostate after removal.

${ }^{\mathrm{b}}$ Grade $4+5$ combined (\%).

recurrence group did not show any significant difference in cytoplasmic $(P=0.079)$ or stromal KLLN expression $(P=0.36)$ (Table 2$)$. The median prostate weight was similar in the relapse-free $(43.00 \pm 5.00 \mathrm{~g})$ and recurrence groups $(43.00 \pm 4.62 \mathrm{~g}, P=0.99)$. The recurrence group had slightly higher preoperative PSA levels $(12.65 \pm 2.09 \mathrm{ng} / \mathrm{ml})$ and larger index tumor volume $\left(4.70 \pm 0.94 \mathrm{~cm}^{3}\right)$ compared with the relapse-free group $(10.75 \pm 3.91 \mathrm{ng} / \mathrm{ml}$ and $4.0 \pm$ $0.84 \mathrm{~cm}^{3}$ respectively), but the differences were not significant ( $P=0.39$ and $P=0.29$ respectively) (Table 2$)$. In the recurrence group, 36\% had SVI compared with $10 \%$ in the relapse-free group $(P=0.002)$ (Table 2$)$. The tumors in the recurrence group also displayed more capsular penetration (51\%) and positive SM (31\%), compared with tumors from the relapse-free group $(6 \%, P<0.0001$ and $6 \%$, $P=0.0012$ respectively) (Table 2 ). Among the eight morphologic variables in our study, loss of nuclear KLLN

Published by Bioscientifica Ltd. 


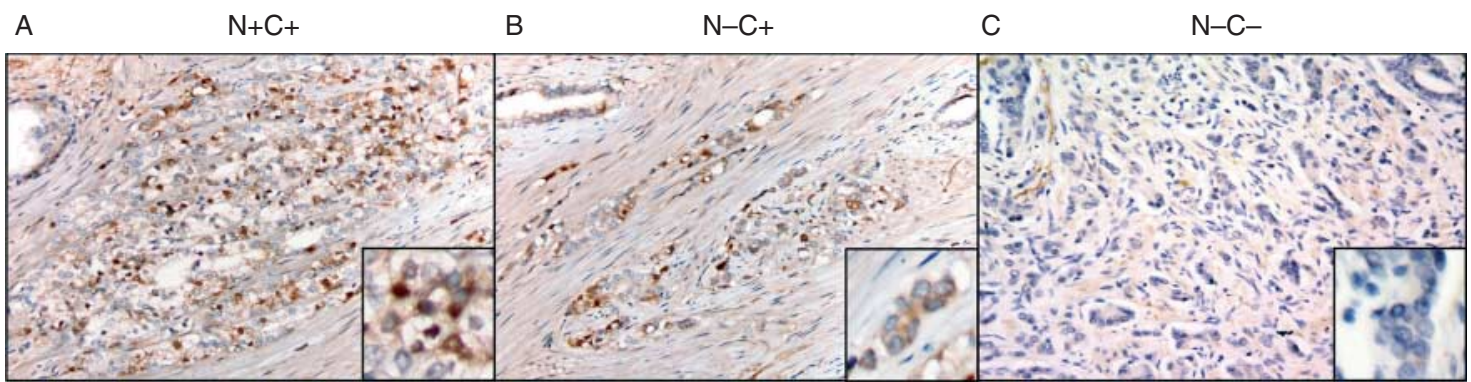

\section{Figure 1}

(A) Positive nuclear and cytoplasmic $(\mathrm{N}+\mathrm{C}+) \mathrm{KLLN}$ immunohistochemical staining in high-grade prostatic adenocarcinoma. (B) Negative nuclear and positive cytoplasmic $(\mathrm{N}-\mathrm{C}+$ ) KLLN immunohistochemical staining in

$(\mathrm{OR}=0.04,95 \%$ CI $0.004-0.49, P=0.012), \mathrm{SVI}(\mathrm{OR}=0.16$, 95\% CI 0.039-0.64, $P=0.010)$, and capsular penetration $(\mathrm{OR}=0.13,95 \%$ CI $0.034-0.50, P=0.003)$ are negatively correlated with patients' RFS (Table 3). Spearman's rank correlation analysis showed that these three factors are not correlated, suggesting nuclear-KLLN expression lost as an independent risk factor in association with RP prognosis for tumor recurrences (Supplementary Table S1, see section on supplementary data given at the end of this article).

\section{Positive nuclear KLLN expression in CaP associates with good prognosis after RP}

We then compared clinical and pathological characteristics in patients with nuclear KLLN-positive tumors and those who lost KLLN expression in tumor nuclei. high-grade prostatic adenocarcinoma. (C) Negative nuclear and cytoplasmic $(\mathrm{N}-\mathrm{C}-$ ) KLLN immunohistochemical staining in high-grade prostatic adenocarcinoma.

Nuclear KLLN expression was detected in 13/109 (12\%) patients (Table 4). We noticed that all nuclear KLLNpositive patients express KLLN in the tumor cytoplasm as well. Patients in this nuclear KLLN-positive group had significantly lower preoperative PSA levels (8.35 \pm $2.45 \mathrm{ng} / \mathrm{ml}$ ) compared with those of the nuclear KLLNnegative group (12.24 $\pm 2.37 \mathrm{ng} / \mathrm{ml}, P=0.031)$ (Table 4$)$. In addition, tumor volume in nuclear KLLN-positive patients $\left(2.66 \pm 0.51 \mathrm{~cm}^{3}\right)$ was significantly smaller compared with that in the nuclear KLLN-negative group $\left(4.61 \pm 0.71 \mathrm{~cm}^{3}\right.$, $P<0.0001$ ) (Table 4). Patients in nuclear KLLN-positive groups have less SVI and positive SM, but the differences are not significant $(P=0.15$ and 0.06 respectively) (Table 4). Of 96 nuclear KLLN-negative tumors, 34\% had capsular penetration, which was not observed in any of the nuclear KLLN-positive tumors $(P=0.011)$ (Table 4$)$.

Table 2 Comparison of patients with recurrent or relapse-free cancer

\begin{tabular}{l} 
Cases \\
\hline Sample size \\
Nuclear KLLN lost \\
Cytoplasmic KLLN lost \\
Stromal KLLN lost \\
Age (years) \\
PSA (ng/ml) \\
Prostate weight $(\mathrm{g})^{\mathrm{a}}$ \\
Total tumor volume $\left(\mathrm{cm}^{3}\right)$ \\
Index tumor volume $\left(\mathrm{cm}^{3}\right)$ \\
Secondary tumor volume $\left(\mathrm{cm}^{3}\right)$ \\
Grade $4 / 5 \%{ }^{\mathrm{b}}$ \\
SVI \\
Capsular penetration \\
Positive margins \\
Days of follow-up \\
Time to failure (days)
\end{tabular}

\begin{tabular}{c}
\hline Relapse-free cancer \\
\hline$n=50$ \\
$38 / 50(76 \%)$ \\
$3 / 50(6 \%)$ \\
$0 / 50(0 \%)$ \\
$63.24 \pm 1.73$ \\
$10.75 \pm 3.91$ \\
$43.00 \pm 5.00$ \\
$4.0 \pm 0.84$ \\
$3.59 \pm 0.81$ \\
$0.39 \pm 0.22$ \\
$50.5 \pm 4.32$ \\
$5 / 50(10 \%)$ \\
$3 / 50(6 \%)$ \\
$3 / 50(6 \%)$ \\
$2251 \pm 161$ \\
NA
\end{tabular}

\begin{tabular}{c}
\hline Recurrent \\
$n=59$ \\
$58 / 59(98 \%)$ \\
$10 / 59(17 \%)$ \\
$1 / 59(2 \%)$ \\
$63.2 \pm 1.55$ \\
$12.65 \pm 2.09$ \\
$43.00 \pm 4.62$ \\
$4.70 \pm 0.94$ \\
$4.30 \pm 0.95$ \\
$0.36 \pm 0.18$ \\
$53.30 \pm 3.93$ \\
$21 / 59(36 \%)$ \\
$30 / 59(51 \%)$ \\
$18 / 59(31 \%)$ \\
$1962 \pm 289$ \\
$198 \pm 55$
\end{tabular}

\begin{tabular}{c}
\hline $\boldsymbol{P}$ value \\
\hline $\mathbf{3 . 4} \times \mathbf{1 0}^{-\mathbf{4}}$ \\
0.079 \\
0.36 \\
0.93 \\
0.39 \\
0.99 \\
0.29 \\
0.28 \\
0.86 \\
0.35 \\
$\mathbf{0 . 0 0 2}$ \\
$\mathbf{3 . 8} \times \mathbf{1 0}$ \\
$\mathbf{0 . 0 0 1 2}$ \\
0.11 \\
$\mathrm{NA}$
\end{tabular}

SVI, seminal vesicle invasion. Bold indicates statistical significance $(P<0.05)$. a Weight of prostate after removal.

${ }^{\mathrm{b}}$ Grade $4+5$ combined (\%).

http://erc.endocrinology-journals.org DOI: 10.1530/ERC-14-0148
(C) 2014 Society for Endocrinology Printed in Great Britain
Published by Bioscientifica Ltd. 
Table 3 Logistic regression model of characteristics associated with RFS in moderate-risk CaP

\begin{tabular}{l} 
Variables \\
\hline Age \\
PSA \\
Prostate weight \\
Index tumor volume \\
Secondary tumor volume \\
Total tumor volume \\
Days of follow-up \\
Nuclear-KLLN lost \\
Cytoplasmic-KLLN lost \\
Stromal-KLLN lost \\
SVI \\
Positive margins \\
Capsular penetration
\end{tabular}

\begin{tabular}{|c|c|c|}
\hline \multicolumn{3}{|c|}{ Univariate } \\
\hline$P$ value & OR & $95 \% \mathrm{Cl}$ \\
\hline 0.29 & 0.96 & $0.88-1.04$ \\
\hline 0.89 & 1.00 & $0.95-1.06$ \\
\hline 0.53 & 1.01 & $0.98-1.04$ \\
\hline 0.78 & 1.34 & $0.18-10.03$ \\
\hline 0.67 & 1.60 & $0.18-14.15$ \\
\hline 0.63 & 0.61 & $0.08-4.60$ \\
\hline 0.50 & 1.00 & $1.00-1.00$ \\
\hline 0.012 & 0.04 & $0.004-0.49$ \\
\hline 0.88 & 1.17 & $0.15-8.79$ \\
\hline 0.14 & 0.28 & $0.05-1.51$ \\
\hline 0.010 & 0.16 & $0.04-0.64$ \\
\hline 0.66 & 1.44 & $0.28-7.31$ \\
\hline 0.003 & 0.13 & $0.03-0.50$ \\
\hline
\end{tabular}

\begin{tabular}{|c|c|c|}
\hline \multicolumn{3}{|c|}{ Multivariate } \\
\hline$P$ value & OR & $95 \% \mathrm{Cl}$ \\
\hline 0.29 & 1.05 & $0.96-1.14$ \\
\hline 0.89 & 1.00 & $0.94-1.05$ \\
\hline 0.53 & 0.99 & $0.96-1.02$ \\
\hline 0.78 & 0.75 & $0.10-5.58$ \\
\hline 0.67 & 0.62 & $0.07-5.51$ \\
\hline 0.63 & 1.64 & $0.22-12.31$ \\
\hline 0.50 & 1.00 & $1.00-1.00$ \\
\hline 0.012 & 0.04 & $0.004-0.49$ \\
\hline 0.88 & 1.17 & $0.15-8.79$ \\
\hline 0.14 & 0.28 & $0.05-1.51$ \\
\hline 0.010 & 0.16 & $0.04-0.64$ \\
\hline 0.66 & 1.44 & $0.28-7.31$ \\
\hline 0.003 & 0.13 & $0.034-0.50$ \\
\hline
\end{tabular}

OR, odds ratio; SVI, seminal vesicles invasion. Bold indicates statistical significance $(P<0.05)$. a Weight of prostate after removal.

Most importantly, only 7\% of nuclear KLLN-positive patients had PSA-defined recurrence compared with $60 \%$ of patients with nuclear KLLN-negative cancers, who had median relapse time of 6.6 months $(P=0.0003)$ (Table 4). Kaplan-Meier plots show that nuclear KLLN-positive patients have significantly improved RFS compared with that of the KLLN-negative group during the average 6.1 years of follow-up $(P=0.002)$ (Fig. 2A). Patients maintaining only cytoplasmic KLLN expression did not show an improved RFS compared with cytoplasmic KLLN-negative patients $(P=0.25)$ (Fig. 2B). In summary, our data suggest that maintaining nuclear KLLN expression in high Gleason score tumors might associate with improved RFS in IRPC patients.

\section{KLLN deletion associates with PTEN deletion in IRPC}

To determine whether loss of KLLN in IRPC is due to genomic deletion, we used the TCGA CaP dataset to analyze $K L L N$ mRNA expression and $K L L N$ putative copy number variation. Among the 343 cases in the Prostate Adenocarcinoma (PRAD) cohort, 244 cases contain clinical and pathology information. We identified 119 patients with Gleason $7 \mathrm{CaP}$. Among the 119 samples, 23 (19\%) have homozygous KLLN deletion and 12 (10\%) have hemizygous KLLN deletion (Fig. 3A). Deletions of $K L L N$ significantly associated with decreased KLLN mRNA expression levels in those tumors $(P<0.0001)$ (Fig. 3A). PTEN deletion was commonly found in advanced CaP. As

Table 4 Comparison of patients with nuclear-KLLN positive or negative tumors

\begin{tabular}{|c|c|c|c|}
\hline Nuclear KLLN expression & Positive & Negative & $P$ value \\
\hline Sample size & $n=13$ & $n=96$ & \\
\hline Age (years) & $60.46 \pm 3.29$ & $63.55 \pm 1.22$ & 0.10 \\
\hline PSA (ng/ml) & $8.35 \pm 2.45$ & $12.24 \pm 2.37$ & 0.031 \\
\hline Prostate weight $(\mathrm{g})^{a}$ & $44.53 \pm 11.54$ & $48.32 \pm 3.51$ & 0.55 \\
\hline Index tumor $\left(\mathrm{cm}^{3}\right)$ & $2.56 \pm 0.55$ & $4.17 \pm 0.71$ & 0.001 \\
\hline Secondary tumors $\left(\mathrm{cm}^{3}\right)$ & $0.10 \pm 0.15$ & $0.42 \pm 0.15$ & 0.007 \\
\hline Total tumor volume $\left(\mathrm{cm}^{3}\right)$ & $2.66 \pm 0.51$ & $4.61 \pm 0.71$ & $4.1 \times 10^{-5}$ \\
\hline Grade $4 / 5 \%$ b & $53.07 \pm 9.18$ & $51.88 \pm 3.01$ & 0.81 \\
\hline SVI & $1 / 13(7 \%)$ & $25 / 96(26 \%)$ & 0.15 \\
\hline Capsular penetration & $0 / 13(0 \%)$ & $33 / 96(34 \%)$ & 0.011 \\
\hline Positive margins & $0 / 13(0 \%)$ & $21 / 96(22 \%)$ & 0.06 \\
\hline Days of follow-up & $2338 \pm 371$ & $2062 \pm 521$ & 0.21 \\
\hline Tumor recurrence & $1 / 13(7 \%)$ & $58 / 96(60 \%)$ & $3.4 \times 10^{-4}$ \\
\hline Time to failure (days) & $\mathrm{NA}^{\mathrm{c}}$ & $280 \pm 56$ & NA \\
\hline
\end{tabular}

SVI, seminal vesicles invasion. Bold indicates statistical significance $(P<0.05)$.

a Weight of prostate after removal.

${ }^{\mathrm{b}}$ Grade $4+5$ combined (\%).

'One recurrent case with relapse at 225 days.

http://erc.endocrinology-journals.org DOI: 10.1530/ERC-14-0148
(C) 2014 Society for Endocrinology Printed in Great Britain
Published by Bioscientifica Ltd. 

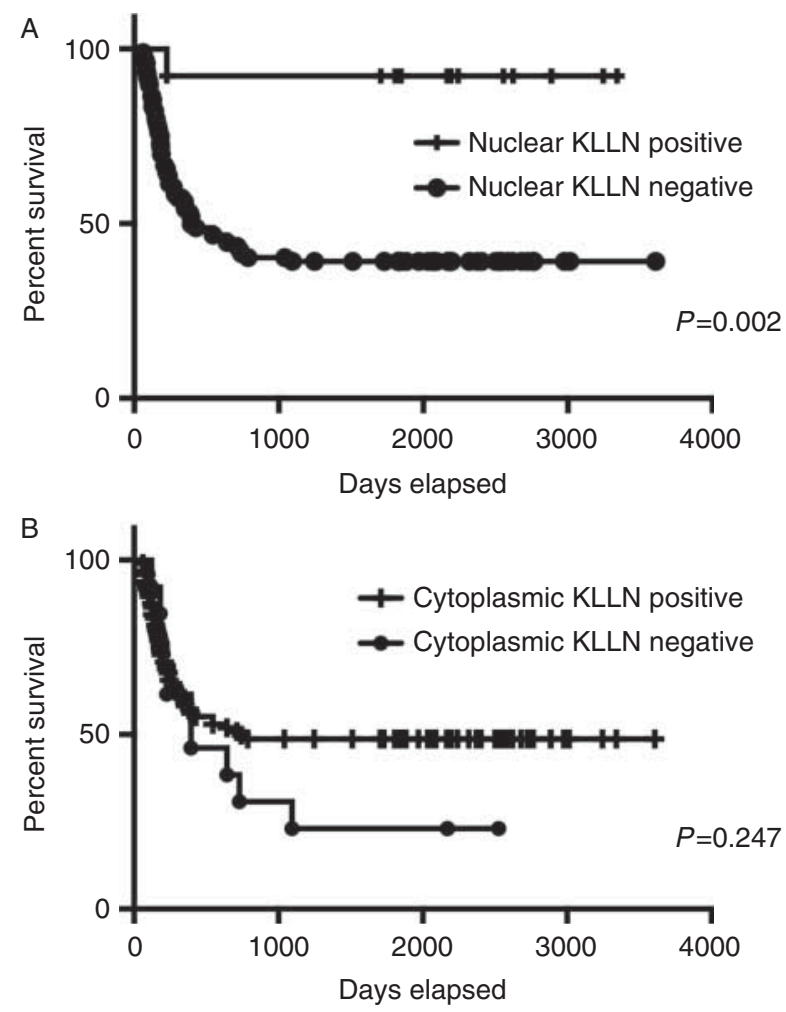

Figure 2

Association between KLLN expression and RFS. (A) Patients with positive nuclear KLLN expression in their prostate carcinomas $(n=13)$ had significantly improved RFS compared with patients with no nuclear KLLN expression ( $n=96, P=0.002$, Mantel-Cox test). (B) Patients with cytoplasmic KLLN-positive tumors ( $n=96$ ) had no significantly improved RFS compared with patients with no cytoplasmic KLLN expression ( $n=13$, $P=0.247$, Mantel-Cox test).

KLLN and PTEN are adjacent to each other and share the same promoter, we then explored whether KLLN deletion in IRPC associates with loss of PTEN. Of the 119 cases, 22 (18\%) have homozygous PTEN deletion, in which 20 cases have homozygous deletions of both KLLN and PTEN (Fig. 3B). Decreased KLLN copy number significantly associated with loss of PTEN in IRPC (Spearman $r=0.91$, $P<0.0001$ ) (Fig. 3C). In addition, the expression of $K L L N$ and PTEN is also associated with IRPC (Fig. 3D). Our analysis shows the high frequency of deletion of both PTEN and KLLN in IRPC, which in turn associates with decrease in both genes' RNA expression.

\section{Discussion}

KLLN was first identified as a p53-targeted DNA-binding protein regulating cell cycle and apoptosis in monkey kidney cells (Cho \& Liang 2008). Our previous study revealed that KLLN is a transcription factor and mainly functions in the cell nucleus (Wang et al. 2013a,b). CaP patients have significantly decreased KLLN expression in the tumor compared with adjacent normal tissue (Wang et al. 2013b). Loss of KLLN expression may be an early event during tumor development and is associated with high Gleason score in CaP (Wang et al. 2013b). In CaP cells, KLLN inhibits AR expression by transcriptional suppression, resulting in decreased PSA levels (Wang et al. 2013b). This function aligns with our current finding that patients with nuclear KLLN-negative tumors have higher preoperative PSA levels, although of course secretion of PSA from normal prostate glands as well as benign prostatic hyperplasia $(\mathrm{BPH})$ contributes to serum PSA levels. In our current cohort, patients with nuclear KLLN-negative tumors have higher prevalence of SVI, positive SM, and capsular penetration. However, Spearman's $\rho$ indicates that nuclear KLLN expression is an independent risk factor for predicting patients' surgical outcome. Patients with grade 4 or 5 malignancies have poorer prognosis than the men with $100 \%$ grade 3 prostate tumors. However, the proportion of grade 4/5 tumors does not distinguish the surgical outcome. Our current study shows that risk of PSA-defined relapse was increased 24-fold in men with nuclear KLLN-negative high-grade tumors, compared with men with nuclear KLLN expression (Table 3). It appears that KLLN expression in the tumor cytoplasm and stroma did not associate with patient pre- or postoperative characteristics or surgical outcome. This suggests that loss of nuclear KLLN expression in neoplasias might play important roles in tumor progression and contribute to tumor aggressiveness. In IRPC patients, preoperation biopsies often only contain grade 3 lesions even though grades 4 or 5 can be observed in the PR specimens. Further validation of our findings in biopsies will provide more evidence for using KLLN as preoperative marker to predictive patient outcome and to personalize postsurgical cancer management.

Genomic deletions of PTEN in CaP have been shown to confer an unfavorable clinical outcome (Basler et al. 2005), most likely through decreased PTEN expression and subsequent upregulation of the AKT signaling pathway (Canby-Hagino et al. 2005, Wang et al. 2010, Costello et al. 2011). Hemizygous and homozygous PTEN deletions were found in $40 \%$ of locally advanced $\mathrm{CaP}$ and the incidence of PTEN deletion in CRPC may reach 70-80\% (Swanson et al. 2006a,b,c, Ramahi et al. 2012). A recent study has shown that, in CaP, the most frequent loss at 10q23 including the PTEN ranges from $36 \mathrm{~kb}$ to $90 \mathrm{Mb}$ in size (Yoshimoto et al. 2012). Considering the fact that $K L L N$ lies in proximity to PTEN in 10q23 and shares a bidirectional promoter with

Published by Bioscientifica Ltd 

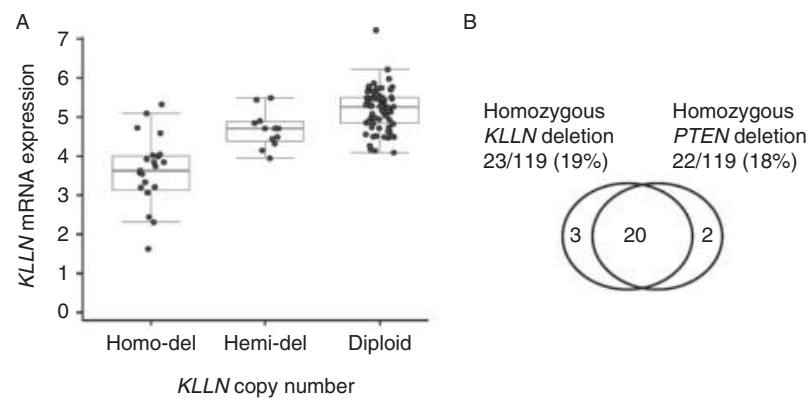

Figure 3

$K L L N$ deletion associated with deletion of PTEN. (A) Box-and -whisker plots showing decreased KLLN mRNA expression levels in tumors with $K L L N$ deletion. Tumors with homozygous KLLN deletion $(n=23)$, hemizygous $K L L N$ deletion $(n=12)$, or diploid-KLLN $(n=84)$ were analyzed. The ends of the box- and -whisker plots represent 5th and 95th percentiles (one-way ANOVA, $P<0.0001$ ). (B) Comparison of IRPC cases with homozygous KLLN

PTEN, such deletions of PTEN in CaP may also include $K L L N$, which may explain the loss of KLLN expression in CaP. Our study revealed that 90\% IRPC tumor samples with homozygous PTEN deletions also have KLLN deletions, indicating that genome insatiability may affect both tumor suppressors simultaneously. As PTEN deletion is commonly found in many types of cancers, it is highly possible that loss of KLLN may play a novel role in the development and progression of other cancer types as well. We have already shown that $K L L N$ expression negatively correlated with $A R$ expression in an independent cohort of CaP from 183 patients (Wang et al. 2013b). AR directly suppresses KLLN transcription in CaP cell lines (Wang et al. 2013b). Therefore, it is also possible that the increased AR activity in advanced CaP results in decreased KLLN expression levels.

$\mathrm{CaP}$ risk drives treatment selection, whereas accurate prediction of surgical outcome for each individual is extremely challenging. A reliable predictive marker for prolonged RFS or PSA failure would be essential for patient cancer management after their surgeries. It could guide the clinician to offer aggressive treatment to patients who are potentially at higher risk. Importantly, it can also prevent overtreatment for high-risk patients who actually have long-term RFS without further intervention. Several previous studies have developed preoperative scoring systems to predict a patient's earlier probability of tumor recurrence (Kattan et al. 1998, San Francisco et al. 2004, Cooperberg et al. 2006, Stephenson et al. 2006). The strength of the current study is the utilization of a single marker to predict the oncologic outcomes for patients with IRPC. To minimize bias and clinical heterogeneity, all our study patients had tumors of the same Gleason score and no one received neoadjuvant therapy before RP or
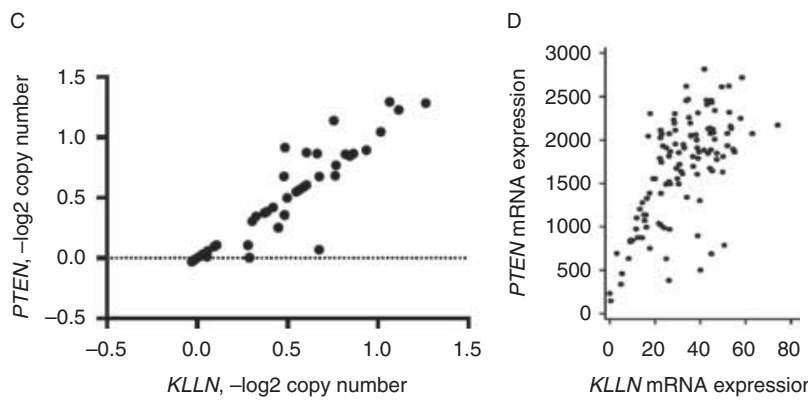

or/and PTEN deletion. (C) Scatter plots showing the association between $K L L N$ and PTEN copy number variation in 119 Gleason 7 IRPC samples (Spearman $r=0.9077, P<0.0001$ ). (D) Scatter plots showing the association between KLLN and PTEN mRNA expression levels in 119 Gleason 7 IRPC samples (Spearman $r=0.60, P<0.05$ ).

adjuvant treatment after RP. We found that patients with nuclear KLLN expression in primary tumors had a high likelihood of long-term RFS despite the presence of high Gleason grade malignancies. In the nuclear KLLN-positive group, only $7 \%$ of the patients experienced PSA failure. In contrast, $60 \%$ of patients who lost nuclear KLLN expression had tumor recurrences, with median relapse time of 6.6 months. Our data also showed that nuclear KLLN expression in primary tumors associates with lower preoperative PSA levels, smaller tumor size, and fewer positive margins. They also suggest that patients with different KLLN expression patterns, regarding subcellular localization, should have different postsurgical cancer management. One limitation in current study is the selection of high-grade tumor lesions, in which majority of cell nuclei are KLLN-negative. Our follow-up study will investigate the association between KLLN expression in Gleason grade 3 tumors and patient surgical outcome.

\section{Supplementary data}

This is linked to the online version of the paper at http://dx.doi.org/10.1530/ ERC-14-0148.

\section{Declaration of interest}

C Eng is a member of the external strategic advisory board of $\mathrm{N}$-of-One and external advisory boards of CareSource and Medical Mutual of Ohio, and an unpaid member of the external scientific advisory boards of GenomOncology and EcoEos. The rest of the authors have no relevant conflicts of interest to declare.

\section{Funding}

This work was supported, in part, by the Susan G Komen for the Cure Foundation (KG101188) and the American Cancer Society Clinical Research Professorship (to C Eng).

Published by Bioscientifica Ltd. 


\section{Author contribution statement}

$C$ Eng and $Y$ Wang developed and designed the concept, and drafted the manuscript. Y Wang, A Roma, R Nolley, F Abdul-Karim, and D M Peehl were involved in the acquisition of data. Y Wang, A Roma, R Nolley, F AbdulKarim, D M Peehl, and C Eng analysed and interpreted the data. C Eng, Y Wang, A Roma, F Abdul-Karim, and D M Peehl were involved in the critical revision of the manuscript. $Y$ Wang performed the statistical analysis. R Nolley and D M Peehl provided administrative, technical, and material support. C Eng supervised the study.

\section{Acknowledgements}

C Eng is the Sondra J and Stephen R Hardis Chair of Cancer Genomic Medicine at the Cleveland Clinic, was a Doris Duke Distinguished Clinical Scientist and is an American Cancer Society Clinical Research Professor, generously funded, in part, by the F.M. Kirby Foundation.

\section{References}

Basler JW, Jenkins C \& Swanson G 2005 Multidisciplinary management of prostate malignancy. Current Urology Reports 6 228-234. (doi:10.1007/ s11934-005-0012-7)

Bennett KL, Campbell R, Ganapathi S, Zhou M, Rini B, Ganapathi R, Neumann HP \& Eng C 2011 Germline and somatic DNA methylation and epigenetic regulation of KILLIN in renal cell carcinoma. Genes, Chromosomes \& Cancer 50 654-661. (doi:10.1002/gcc.20887)

Canby-Hagino ED, Swanson GP, Crawford ED, Basler JW, Hernandez J \& Thompson IM 2005 Local and systemic therapy for patients with metastatic prostate cancer: should the primary tumor be treated? Current Urology Reports 6 183-189. (doi:10.1007/s11934-005-0006-5)

Cho YJ \& Liang P 2008 Killin is a p53-regulated nuclear inhibitor of DNA synthesis. PNAS 105 5396-5401. (doi:10.1073/pnas.0705410105)

Cooperberg MR, Freedland SJ, Pasta DJ, Elkin EP, Presti JC Jr, Amling CL, Terris MK, Aronson WJ, Kane CJ \& Carroll PR 2006 Multiinstitutional validation of the UCSF cancer of the prostate risk assessment for prediction of recurrence after radical prostatectomy. Cancer 107 2384-2391. (doi:10.1002/cncr.22262)

Costello LC, Franklin RB, Zou J, Feng P, Bok R, Swanson MG \& Kurhanewicz J 2011 Human prostate cancer ZIP1/zinc/citrate genetic/metabolic relationship in the TRAMP prostate cancer animal model. Cancer Biology \& Therapy 12 1078-1084. (doi:10.4161/cbt.12.12.18367)

D'Amico AV, Whittington R, Malkowicz SB, Schultz D, Blank K, Broderick GA, Tomaszewski JE, Renshaw AA, Kaplan I, Beard CJ et al. 1998a Biochemical outcome after radical prostatectomy, external beam radiation therapy, or interstitial radiation therapy for clinically localized prostate cancer. Journal of the American Medical Association 280 969-974. (doi:10.1001/jama.280.11.969)

D'Amico AV, Whittington R, Malkowicz SB, Schultz D, Kaplan I, Beard CJ, Tomaszewski JE, Renshaw AA, Loughlin KR, Richie JP et al. 1998 b Calculated prostate cancer volume greater than $4.0 \mathrm{~cm} 3$ identifies patients with localized prostate cancer who have a poor prognosis following radical prostatectomy or external-beam radiation therapy. Journal of Clinical Oncology 16 3094-3100.

D'Amico AV, Schnall M, Whittington R, Malkowicz SB, Schultz D, Tomaszewski JE \& Wein A 1998c Endorectal coil magnetic resonance imaging identifies locally advanced prostate cancer in select patients with clinically localized disease. Urology 51 449-454. (doi:10.1016/ S0090-4295(97)00630-4)

D'Amico AV, Whittington R, Malkowicz SB, Fondurulia J, Chen MH, Tomaszewski JE \& Wein A 1998d The combination of preoperative prostate specific antigen and postoperative pathological findings to predict prostate specific antigen outcome in clinically localized prostate cancer. Journal of Urology 160 2096-2101. (doi:10.1016/S00225347(01)62251-1)

DeSantis C, Naishadham D \& Jemal A 2013 Cancer statistics for African Americans, 2013. CA: A Cancer Journal for Clinicians 63 151-166. (doi:10.3322/caac.21173)

Kattan MW, Eastham JA, Stapleton AM, Wheeler TM \& Scardino PT 1998 A preoperative nomogram for disease recurrence following radical prostatectomy for prostate cancer. Journal of the National Cancer Institute 90 766-771. (doi:10.1093/jnci/90.10.766)

Meng MV, Elkin EP, Latini DM, Duchane J \& Carroll PR 2005 Treatment of patients with high risk localized prostate cancer: results from cancer of the prostate strategic urological research endeavor (CaPSURE). Journal of Urology 173 1557-1561. (doi:10.1097/01.ju.0000154610.81916.81)

Park S, Meng MV, Elkin EP, Speight JL, DuChane J \& Carroll PR 2005 Androgen deprivation use with external beam radiation for prostate cancer: results from CaPSURE. Journal of Urology 174 1802-1807. (doi:10.1097/01.ju.0000177089.93728.20)

Ramahi EH, Swanson GP, Jackson MW, Du F \& Basler JW 2012 High-grade prostate cancer: favorable results in the modern era regardless of initial treatment. ISRN Oncology 2012 596029. (doi:10.5402/2012/596029)

San Francisco IF, Regan MM, Olumi AF \& DeWolf WC 2004 Percent of cores positive for cancer is a better preoperative predictor of cancer recurrence after radical prostatectomy than prostate specific antigen. Journal of Urology 171 1492-1499. (doi:10.1097/01.ju.0000118690. 05943.c0)

Siegel R, Naishadham D \& Jemal A 2013 Cancer statistics, 2013. CA: A Cancer Journal for Clinicians 63 11-30. (doi:10.3322/caac.21166)

Stephenson AJ, Scardino PT, Eastham JA, Bianco FJ Jr, Dotan ZA, Fearn PA \& Kattan MW 2006 Preoperative nomogram predicting the 10-year probability of prostate cancer recurrence after radical prostatectomy. Journal of the National Cancer Institute 98 715-717. (doi:10.1093/jnci/djj190)

Swanson G, Thompson I, Basler J \& Crawford ED 2006a Metastatic prostate cancer-does treatment of the primary tumor matter? Journal of Urology 176 1292-1298. (doi:10.1016/j.juro.2006.06.069)

Swanson GP, Thompson IM \& Basler J 2006b Current status of lymph node-positive prostate cancer: incidence and predictors of outcome. Cancer 107 439-450. (doi:10.1002/cncr.22034)

Swanson GP, Thompson IM \& Basler J 2006c Treatment options in lymph node-positive prostate cancer. Cancer 106 2531-2539. (doi:10.1002/ cncr.21947)

Wang Y, Romigh T, He X, Orloff MS, Silverman RH, Heston WD \& Eng C 2010 Resveratrol regulates the PTEN/AKT pathway through androgen receptor-dependent and -independent mechanisms in prostate cancer cell lines. Human Molecular Genetics 19 4319-4329. (doi:10.1093/hmg/ ddq354)

Wang Y, He X, Yu Q \& Eng C 2013a Androgen receptor-induced tumor suppressor, KLLN, inhibits breast cancer growth and transcriptionally activates p53/p73-mediated apoptosis in breast carcinomas. Human Molecular Genetics 22 2263-2272. (doi:10.1093/hmg/ddt077)

Wang Y, Radhakrishnan D, He X, Peehl DM \& Eng C 2013b Transcription factor KLLN inhibits tumor growth by AR suppression, induces apoptosis by TP53/TP73 stimulation in prostate carcinomas, and correlates with cellular differentiation. Journal of Clinical Endocrinology and Metabolism 98 E586-E594. (doi:10.1210/jc.2012-3490)

Yoshimoto M, Ludkovski O, DeGrace D, Williams JL, Evans A, Sircar K, Bismar TA, Nuin P \& Squire JA 2012 PTEN genomic deletions that characterize aggressive prostate cancer originate close to segmental duplications. Genes, Chromosomes \& Cancer 51 149-160. (doi:10.1002/ gcc.20939)

Received in final form 19 May 2014 Accepted 21 May 2014 http://erc.endocrinology-journals.org DOI: 10.1530/ERC-14-0148
(C) 2014 Society for Endocrinology Printed in Great Britain 